\title{
APTIDÃO FíSICA, ESTUDO DE ALUNOS DO 3 CICLO DOS CONCELHOS DE TORRE DE MONCORVO E VILA NOVA DE GAIA
}

\author{
PHYSICAL EDUCATION, STUDY OF 3 THE EDUCATION IN \\ THE TOWNS OF TORRE DE MONCORVO AND VILA NOVA DE GAIA
}

\author{
AUTORES \\ Ruben Gonçalves Pereira' \\ José Luís Soidán \\ 'Universidade de Vigo
}

APTIDÃo FísICA, ESTUDO DE ALUNOS DO 3 CICLO DOS CONCELHOS DE TORRE DE MONCORVO E VILA NOVA DE GAIA

4(1): 75-81

\section{PALAVRAS-CHAVE}

aptidão física; estudo comparativo; rural/urbano.

\section{KEYWORDS}

physical fitness; comparative studies; rural/urban.

data de submissão

Dezembro 2007

data de aceitação

Março 2008
RESUMO

O objectivo deste estudo que nos propusemos realizar, orientou-se fundamentalmente para a tentativa de responder a um conjunto de questões pertinentes: A interioridade será um factor limitativo para o desenvolvimento harmonioso do jovem, ao nível da Aptidão Física? Os perfis da aptidão física são distintos quanto ao nível em função da Interioridade/Litoralidade? Para isso desenvolvemos um estudo em que caracterizamos e avaliamos alunos de ambos os sexos, do 2 으 e 3 응 ciclos do ensino básico, de duas zonas diametralmente opostas de Portugal: uma do litoral citadino, Vila Nova de Gaia, outra do interior profundo, Torre de Moncorvo, e investigamos a existência de interferências nos níveis de aptidão física dos alunos.

Este estudo exploratório configurou-se a partir de uma amostra do tipo aleatória por aglomerados compos-

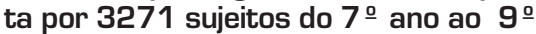
ano de escolaridade. A bateria de testes aplicada foi a designada de "FACDEX" - Desenvolvimento Somato-Motor e factores de Excelência Desportiva na População Portuguesa. (Marques et al., 1991), obtendo-se uma análise detalhada dos níveis de Aptidão Física dos jovens dos 10 aos 17 anos de idade.

Os resultados permitiram concluir que: Os alunos da Zona Rural apresentam um padrão ligeiramente superior nos testes de Força Superior, "Bola 2Kg"; Força Média "Sit Up's" e Força Inferior "Salto em Comprimento".

Os alunos da Zona Urbana apresentam valores padrão superiores no teste de Flexibilidade "Sit and Reach"; Força Superior "Lançamento de bola de Hóquei"; "Dinamometria"; Agilidade "10x5 Mts." e "Resistência 12'". Relativamente à Velocidade, apresentam valores idênticos.

Os alunos da Zona Urbana apresentam valores de peso superior por idade no sexo masculino e feminino, com excepção dos 14 a 16 anos do sexo masculino, e a partir dos 15 anos nas raparigas.

As raparigas do Meio Rural apresentam Índices de Massa Corporal Superiores em todos os anos lectivos com excepção do 8 ํ Ano.

\section{ABSTRACT}

The aim of this study that we proposed to develop was fundamentally guided as an attempt to answer a group of relevant questions:

Is living in the interior of a country a restraining factor for the harmonious physical, mental and social development of the youth?

Are physical aptitudes profiles distinct in terms of the level, depending on the fact that young people live in the interior or in the coast of a country? To answer these questions we develop a study in which we characterize and evaluate students of both sexes, from the $2^{\text {nd }}$ and $3^{\text {rd }}$ cycles of the Basic Education (from $5^{\text {th }}$ to $9^{\text {th }}$ grade), from schools of two diametrical opposed areas of Portugal: one school in a city by the coast - Vila Nova de Gaia, the other in the deep interior of Portugal Torre de Moncorvo and we search the existence or not of an influence on the students' levels of physical aptitudes. This exploratory study was made from a random sample composed of 3271 subjects from the $5^{\text {th }}$ grade to the $\mathbf{9}^{\text {th }}$ school grades. The battery of tests applied were the ones designated as "FACDEX" - Somato-Motor Development and Sports Excellence factors in the Portuguese Population. (Marques et al., 1991), obtaining a detailed analysis of the levels of Physical Aptitudes of young people from 10 to 17 years old. The results allowed us to conclude that the students of the Rural areas present a slightly higher standard in the Upper strength tests (2 kg ball), Medium strength (Sit Up's] and Lower strength (Long jump).

The students of the Urban Zone present higher standard levels in the Flexibility tests (Sit and Reach), Upper strength (Hockey ball Launching"); "Dinamometry"; quickness "10x5 Metres." and "Resistance 12". In terms of Speed, they show identical values.

The students of the Urban Zone show higher weight values in terms of age in the female and male sex, with the exception of male individuals from the 14 to 16 years old and the girls after 15 years old.

The girls of the Rural Environment show superior body mass rates during all school grades, with the exception of the $8^{\text {th }}$ grade. 


\section{INTRODUC̣ÃO}

Actualmente em pleno século XXI, a Actividade Física, e as questões a ela associada, tem vindo a crescer de interesse e a estar na moda.

A preocupação com a prática de actividade física, associada à saúde tem vindo a tornar-se de grande relevo e importância, no âmbito dos estudos e investigações nas ciências do desporto.

Borms (1988) refere que milhões de pessoas praticam actividade física de forma mais ou menos regular e sistemática ou, pelo menos, assim o afirmam.

Das afirmações anteriores, torna-se pertinente inferir que a escola como local de eleição para o desenvolvimento do processo ensino-aprendizagem de todo e qualquer ser humano, relativamente ao desenvolvimento de estratégias de educação para a saúde, é o sítio por excelência.

A aula de educação física, inevitavelmente, tem que promiscuir-se e assumir a sua cota parte de responsabilidade, que é grande, na promoção da aptidão física referenciada à saúde, e na criação de hábitos de vida Fisicamente activos e dinâmicos. Paralelamente, sabe-se que o desenvolvimento de hábitos, comportamentos e atitudes descritoras de estilos de vida saudáveis e activos tendem a desenvolver-se cedo, no seio familiar e que prosseguem na escola. Daí, provavelmente decorre a crença, muito presente em profissionais de Educação Física [EF], que a infância e a adolescência possam representar períodos óptimos para a intervenção pedagógica no sentido de estimular hábitos e comportamentos que se espera manter-se durante toda a vida.

0 presente estudo, tratou-se de definir e caracterizar com objec- tividade as populações alvo. Tratou-se portanto de um estudo descritivo-exploratório (survey), os níveis de aptidão física, referenciados aos alunos do $2^{\circ}$ e $3^{\circ}$ Ciclos de Ensino, dos Concelhos de Torre de Moncorvo, Distrito de Bragança, e Vila Nova de Gaia, Distrito do Porto.

A ausência de qualquer estudo nesta área de Portugal, contrapondo com zonas diametralmente opostas pela sua natureza geográfica, acessibilidade e ofertas de produtos serviços e bens, imbuiu-nos de um espírito de conquista de conhecimento.

Malina (1994) salienta que as componentes da aptidão física são influenciadas pelo processo individual de crescimento associado à maturação e não apenas pelo estímulo da prática de actividade física regular. Maia (1991), cit. refere a este propósito, que "a idade cronológica é um indicador temporal extremamente falacioso, dado que não é sensível à variação evidenciada pelos jovens atletas do mesmo intervalo etário” (pág. 23).

\section{OBJECTIVO}

0 tipo de investigação que nos propusemos realizar, orientou-se fundamentalmente para uma caracterização e avaliação em alunos de ambos os sexos, do 2 ㅇ e $3^{\circ}$ ciclos do ensino básico, de dois Concelhos de zonas diametralmente opostas de Portugal. Um do Litoral citadino, Vila Nova de Gaia e outro do Interior profundo, Torre de Moncorvo.

\section{Objectivos especificos}

1. Conhecer, descrever e comparar o desempenho motor ao nível da coordenação motora e aptidão física associada à saúde nos dois meios distintos.
2. Dotar a Direcção Regional Norte, os Concelhos e concretamente as escolas, de informações precisas relativamente a este domínio.

\section{Justificação da Bateria adoptada [FACDEX]}

A bateria de testes aplicada foi a designada de "FACDEX" - Desenvolvimento Somato-Motor e factores de Excelência Desportiva na População Portuguesa. (Marques et al., 1991).

Adoptamos esta bateria por razões de funcionalidade, numa perspectiva de comparar dados de outros trabalhos científicos realizados em Portugal.

\section{METODOLOGIA}

\section{Caracterização da Amostra}

Este estudo exploratório configurou-se a partir de uma amostra do tipo aleatória por aglomerados composta por 3271 sujeitos do $5 \stackrel{0}{ }$ ano de escolaridade ao $9^{\circ}$ ano de escolaridade.

\section{Dimensão e critérios de selecção da amostra}

0 presente estudo realizou-se em Concelhos distintos: Torre de Moncorvo, distrito de Bragança, Vila Nova de Gaia, distrito do Porto.

A escolha das escolas obedeceu a critérios de localização geográfica e representatividade equitativa de idades, género e ambiente-sócio-económico. 
As escolas do meio urbano, mais populosas, situavam-se nos centros e pertencem a freguesias consideradas exclusivamente como urbanas, assim como as de Torre de Moncorvo, exclusivamente rurais, segundo o critério da classificação territorial do Instituto Nacional de Estatística (INE, 2003).

Os critérios de inclusão que estiveram subjacentes à selecção das turmas e alunos objecto do estudo foram:

- Serem turmas de 2 o ciclo do ensino Básico ( 5ㅇ e 6으 Ano escolaridade).

- Serem turmas de 3 Ciclo do ensino básico $\left[7^{\circ}, 8^{\circ}\right.$ e $9^{\circ}$ Ano de escolaridade].

- Serem leccionadas por professores de Educação Física Licenciados;

- Só participarem os alunos que tenham autorização da[o] Encarregado de Educação e livremente aceitem.

Os critérios de exclusão que estiveram subjacentes à selecção das turmas objecto de estudo foram:

- Os alunos com necessidades Educativas Especiais, foram excluídos pelos motivos associados aos aspectos intrínsecos de que padecem;

- Os alunos que de uma forma muito evidente não participem activamente na realização das tarefas propostas;

- Os alunos que apresentem em algumas pregas de adiposidade valores não fiáveis por dificuldades da sua medição;

- Os alunos que não cumpram todas as tarefas propostas;

- Os alunos que apresentem dificuldades pontuais de saúde e que limitem a sua execução, nomeadamente uma Gripe, Constipação.

\begin{tabular}{|c|c|c|}
\hline Testes & Objectivos & Capacidades \\
\hline Sit and Reach & $\begin{array}{l}\text { Mobilidade da coluna vertebral e tensão dos } \\
\text { músculos dorso-lombares e ísquio-tiniais }\end{array}$ & $\begin{array}{l}\text { Mobilidade } \\
\text { articulo-ligamentar }\end{array}$ \\
\hline Corrida de $50 \mathrm{~m}$. & Velocidade de corrida & Velocidade \\
\hline $\begin{array}{l}\text { Arremesso } \\
\text { peso } 20 \mathrm{Kg}\end{array}$ & Força explosiva dos membros superiores & Força superior \\
\hline $\begin{array}{l}\text { Lançamento bola } \\
\text { Hóquei em campo }\end{array}$ & Força explosiva dos membros superiores & Força superior \\
\hline $\begin{array}{l}\text { Salto em compri- } \\
\text { mento sem corrida } \\
\text { preparatória }\end{array}$ & Força explosiva dos membros inferiores & Força inferior \\
\hline $10 \times 5$ metros & Agilidade [Coordenação/Velocidade] & $\begin{array}{l}\text { Coordenação } \\
\text { oculo-pedal }\end{array}$ \\
\hline $\begin{array}{l}\text { Dinamometria } \\
\text { manual }\end{array}$ & $\begin{array}{l}\text { Força máxima estática dos músculos } \\
\text { de preensão }\end{array}$ & Força estática \\
\hline Sit Up's - 60” & Força - Resistência dos músculos abdominais & Força abdominal \\
\hline Corrida 12' & $\begin{array}{l}\text { Capacidade de Resistência de média duração. } \\
\text { Economia do sistema cardio-respiratório }\end{array}$ & Resistência \\
\hline
\end{tabular}

TABELA1

Estrutura da bateria de testes referenciados por objectivos e capacidades.

\begin{tabular}{|c|c|c|c|c|}
\hline \multicolumn{2}{|c|}{ Rural } & \multicolumn{2}{|c|}{ Urbana } & \multirow{2}{*}{$\begin{array}{l}\text { Total } \\
495\end{array}$} \\
\hline $5^{\circ}$ ano & 73 & $5^{\circ}$ ano & 422 & \\
\hline $6^{\circ}$ ano & 80 & 6음 ano & 389 & 469 \\
\hline $7^{\circ}$ ano & 79 & $7 \stackrel{\circ}{\text { ano }}$ & 656 & 735 \\
\hline $8^{\circ}$ ano & 89 & $8^{\circ}$ ano & 741 & 830 \\
\hline 9ㅇ ano & 48 & 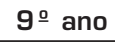 & 694 & 742 \\
\hline TOTAL & \multicolumn{2}{|l|}{369} & 2902 & 3271 \\
\hline
\end{tabular}

TABELA?

Representação da amostra do estudo por anos de escolaridade.

\begin{tabular}{ccc|cc|c} 
& \multicolumn{2}{c|}{ RURAL } & \multicolumn{2}{c|}{ URBANA } & Total \\
Idade & Femininos & Masculinos & Femininos & Masculinos & \\
\hline 10 & - & - & 40 & 41 & 81 \\
11 & 17 & 13 & 201 & 206 & 437 \\
12 & 40 & 48 & 113 & 126 & 327 \\
13 & 63 & 77 & 227 & 227 & 594 \\
14 & 25 & 34 & 389 & 419 & 867 \\
15 & 19 & 14 & 258 & 374 & 665 \\
16 & 8 & 11 & 108 & 104 & 231 \\
17 & - & - & 29 & 35 & 64 \\
18 & - & - & 2 & 3 & 5 \\
\hline TOTAL & 172 & 197 & 1367 & 1535 & 3271 \\
\hline
\end{tabular}

Representação da amostra do estudo por idade cronológica. 


\section{INSTRUMENTARIUM}

\section{Aptidão Física}

- 4 Colchões de espuma de polietileno, de densidade igual a $25 \mathrm{Kg} / \mathrm{m}^{3}$, com as seguintes dimensões: $180 \mathrm{~cm}$ de comprimento, $75 \mathrm{~cm}$ de largura e $10 \mathrm{~mm}$ de espessura, feitos em Portugal;

- 4 Cones de sinalização de cor laranja, com base quadrada com $21 \mathrm{~cm}$ de aresta e cone com $30 \mathrm{~cm}$ de altura, base de $17 \mathrm{~cm}$ de diâmetro e topo com $4,5 \mathrm{~cm}$ de diâmetro, feitos na Indonésia;

- 1 Bola de hóquei em campo de marca "Mersion", modelo "popular", de $156 \mathrm{gr}$, com $7 \mathrm{~cm}$ de diâmetro e $23 \mathrm{~cm}$ de perímetro feita no Reino Unido;

- 1 Bola lastrada de marca "Heavymed" de $2000 \mathrm{gr}$, com $15 \mathrm{~cm}$ de diâmetro e $50 \mathrm{~cm}$ de perímetro feita em Itália;

- 2 Réguas de marca "Ramirex", com escala em centímetros, com $50 \mathrm{~cm}$ de comprimento, $4,3 \mathrm{~cm}$ de largura e $2 \mathrm{~mm}$ de espessura, feitas em Portugal;

- 1 Fita métrica metálica de marca “Medid”, referência N. 6283, com escala em centímetro, com 3 metros de comprimento por $16 \mathrm{~mm}$ de largura;

- 1 Fita métrica de marca "Belota", referência N. ํ 50051-15, em polietileno, com escala em centímetros, com 15 metros de comprimento por 15mm de largura, feita em Portugal;

- 1 Dinamómetro de mão, "GRIP-A", feito no Japão;

- 1 Caixa de medição do teste "Sit and Reach" com 28,5cm de altura, $22 \mathrm{~cm}$ de largura por $36,5 \mathrm{~cm}$ de profundidade;

- Fichas de registo individual/ turma;

\section{Organização do trabalho no terreno}

0 trabalho no terreno foi realizado primeiro nas escolas do Concelho de Torre de Moncorvo, local onde me encontrava a leccionar. Seguidamente foi realizado no Concelho de Vila Nova de Gaia, na escola de Almeida Garrett. Os locais para a recolha de dados, foram as próprias escolas, durante os tempos lectivos, em dias acordados com os Concelhos Executivos e os Professores de Educação Física das respectivas turmas. 0 questionário Sócio-económico foi ministrado numa aula teórica de 50', os testes e as medidas antropométricas foram realizados nas aulas práticas. Por outro lado, de forma a realizarmos os testes propostos de igual forma, ou pelo menos, bastante aproximada, optou-se como estratégia a realização dos testes feita pelo mínimo de pessoas, uma, e sempre em cada escola, desde que as condições fossem semelhantes ao nível dos espaços e pisos.

\section{Protocolo de recolha de dados e aplicação dos testes}

1 Recolher o consentimento dos Encarregados de Educação

2ำ Recolher a anuência dos próprios alunos.

3 Aplicação do Questionário numa aula de 50 minutos, numa sala de aula.

4 Recolha de dados Antropométricos

5 Realização de um prévio aquecimento protocolizado para a aplicação da bateria de testes físicos.

6 Aplicação dos testes físicos, com intervalos de recuperação de 10-12' entre cada teste. A ordem de realização dos testes foi:
1 Dia:

- Prova de Agilidade 10x5 mts.

- Lançamento da Bola de Hóquei

- Sit and Reach

- Velocidade 50 mts.

2음ia:

- Dinamometria

- Salto em Comprimento

- Lançamento de Bola de 2Kgs

- Corrida 12'

Antes de cada prova explicou-se o seu objectivo, os critérios de execução, assim como potenciais riscos quando mal executados, favorecendo a compreensão e a adaptação de cada um dos alunos.

Todas as provas foram realizadas da parte da manhã, em dias amenos sem temperaturas extremas, entre os 18 e 22 graus.

Todos os alunos foram avisados pelos seus professores para nos dias da avaliação através dos testes físicos, o seu Pequeno-almoço fosse normal, mas que não comessem excessivamente, pois iria prejudicar a sua prestação nos testes físicos. No momento de avaliar os alunos nos diferentes testes físicos, é preciso realizar um aquecimento prévio, igual para todos os alunos do estudo. Este tem sempre a mesma ordem e a mesma duração dos exercícios.

\section{Protocolo de realização do aquecimento}

Meios:

- Exercícios de mobilidade articular - 5 min.

- Corrida contínua - 10 min.

Conteúdos por ordem:

- Tornozelos: Flexão, extensão, circundução interior e exterior 10x.

- Joelhos: Flexão, extensão circundução interior e exterior 10x.

- Pernas: Elevações frontais e laterais $10 x$. 
- Ancas. Inclinações laterais, frontais e rotações $10 x$.

- Ombros: Circunduções simétricas e assimétricas frontais e de recta-guarda 10x.

- Braços: Flexão, extensão, circundução interior e exterior 10x.

- Cotovelos. Flexão, extensão, circundução interior e exterior 10x.

- Pulsos: Circunduções interiores e exteriores 10x.

- Mãos: Abrir e fechar 10x.

- Cabeça e Pescoço: Torção lateral e frontal e rotações 10x.

- Corrida Contínua 10 min.

\section{Procedimentos estatísticos}

Os procedimentos estatísticos utilizados para determinar a validade dos dados foram: o erro técnico de medida [ETM $^{1}$, o coeficiente de variação $(\mathrm{CV})^{2}$.

Utilizou-se o teste de Kolmogorov-Smirnov, para analisar a normalidade da distribuição.

0 tratamento e análise estatística dos dados foi efectuado no programa estatístico "Statistical Package for Social Sciences" - SPSS (Versão 11.5 para Windows] e incluiu vários procedimentos que serão especificados com maior detalhe, ao longo desta secção.

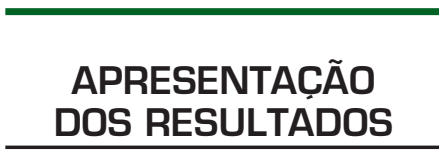

A apresentação dos dados, terá uma estrutura, definida de forma objectiva, onde será feita uma breve análise aos resultados da população da amostra, aos dados relativos às características dos testes da bateria de testes "FACDEX".

Ambos os distritos apresentam valores superiores nos rapazes em relação às raparigas.

\begin{tabular}{lcccc}
\hline & & \multicolumn{2}{c}{ Sexo } \\
Frequency & Percent & $\begin{array}{c}\text { Valid } \\
\text { Percent }\end{array}$ & $\begin{array}{c}\text { Cumulative } \\
\text { Percent }\end{array}$ \\
\hline Valid Rapaz & 37 & 63,8 & 63,8 & 63,8 \\
Rapariga & 21 & 36,3 & 36,3 & 100,0 \\
Total & 58 & 100,0 & 100,0 & \\
\hline
\end{tabular}

TABELA4

Frequência de rapazes e raparigas em V. N. de Gaia.

\begin{tabular}{ccccc}
\hline & & \multicolumn{3}{c}{ Sexo } \\
& Frequency & Percent & $\begin{array}{c}\text { Valid } \\
\text { Percent }\end{array}$ & $\begin{array}{c}\text { Cumulative } \\
\text { Percent }\end{array}$ \\
\hline Valid Rapaz & 59 & 53,2 & 53,2 & 53,2 \\
Rapariga & 52 & 46,8 & 46,8 & 100,0 \\
Total & 111 & 100,0 & 100,0 & \\
\hline & & & & TABELA4 \\
& & Frequência de rapazes e raparigas em Moncorvo.
\end{tabular}

Os alunos de Gaia apresentam valores superiores de flexibilidade, relativamente a Moncorvo, Média de 7,4 contra 3,8 centímetros.

0 concelho de Moncorvo apresenta valores superiores.

A tendência observada no nosso estudo de os alunos de Moncorvo, apresentarem valores superiores de força superior comparativamente aos de Gaia, é confirmado neste teste com médias de 23,4 contra 21,8 metros.

Relativamente a Velocidade, apresentam valores sem diferenças estatisticamente significativas.

0 Meio Rural apresenta valores ligeiramente superiores.
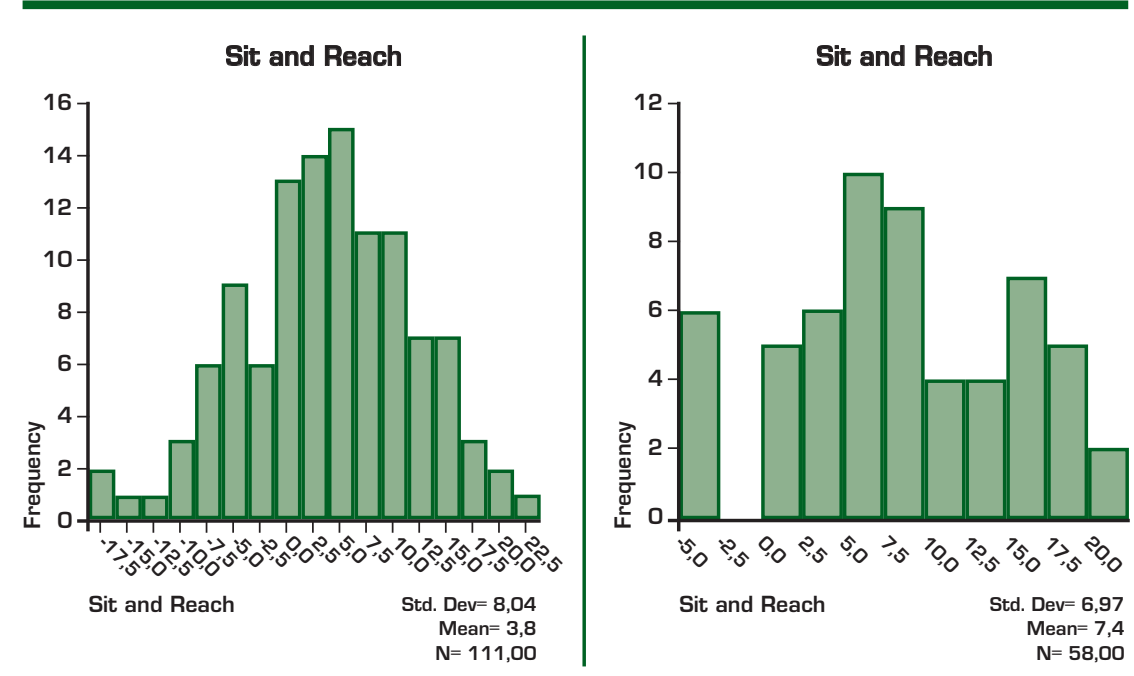

FIGURA1 Comparação "Sit and Reach". 

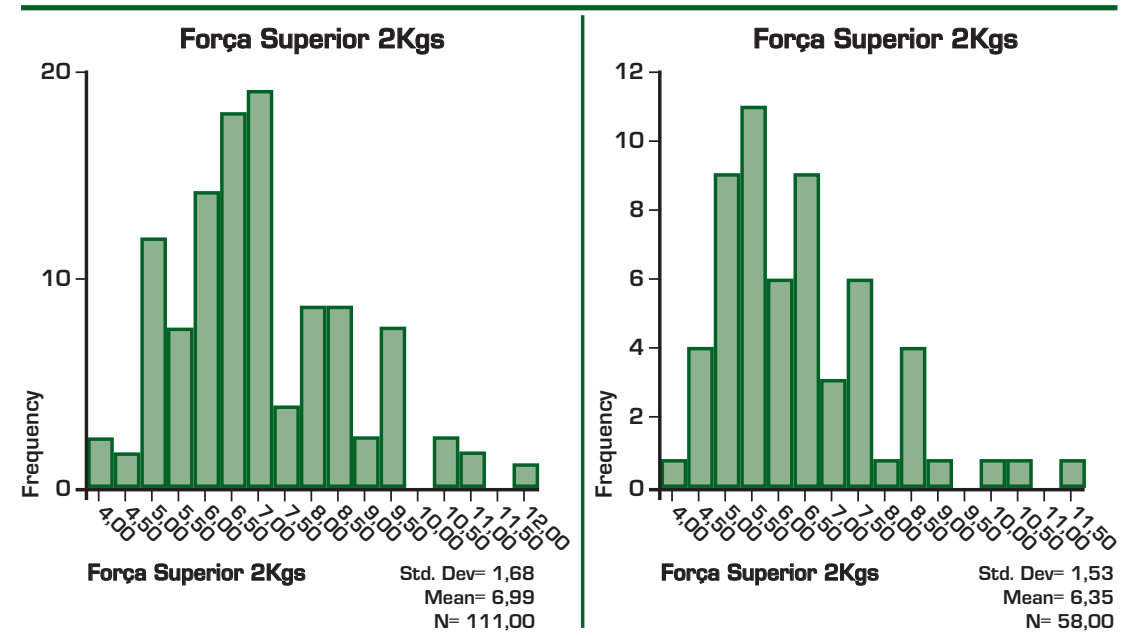

FIGURA2 Comparação de Força superior 2Kg.
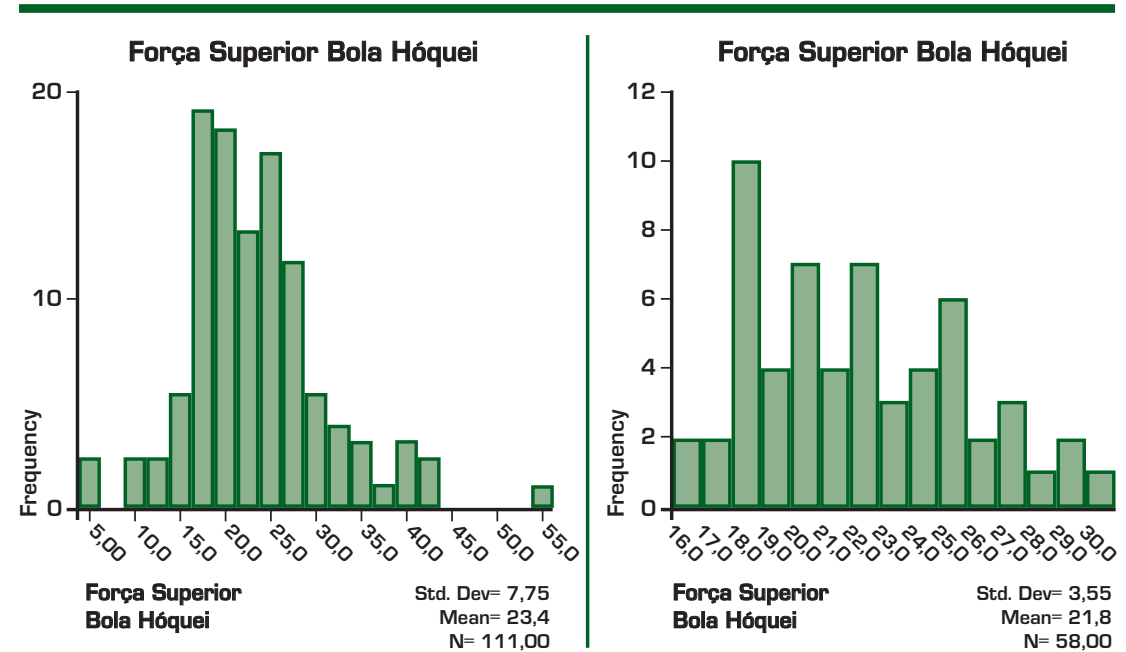

FIGURA3 Comparação de Força superior Bola de Hóquei.
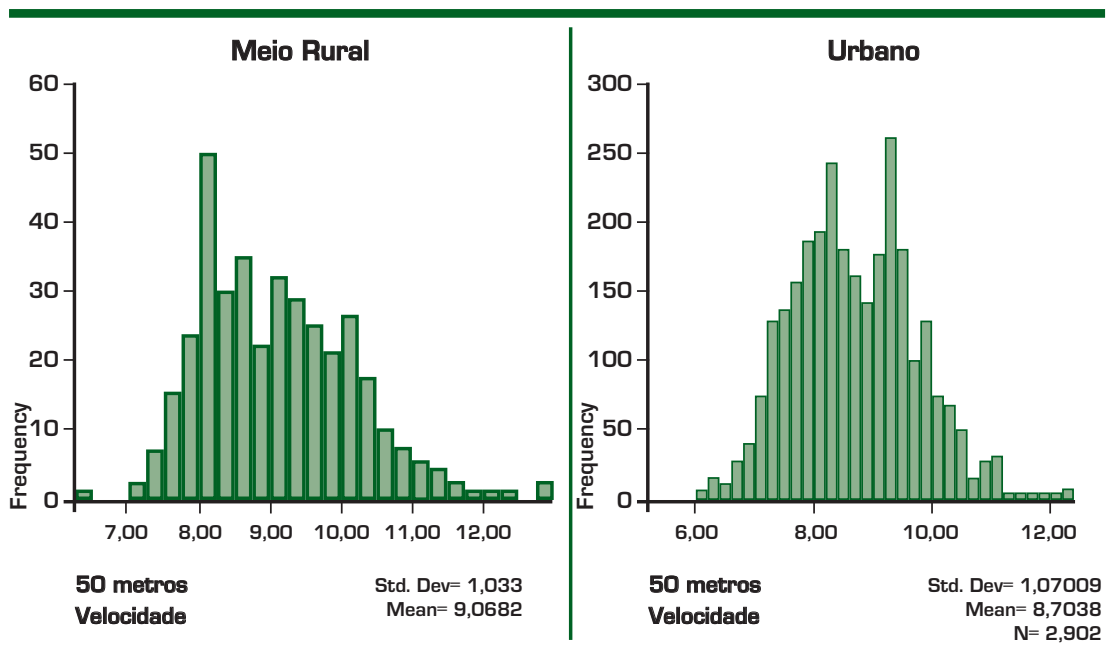

FIGURA4 Comparação de Velocidade 50 Metros.

\section{DISCUSSÃO DOS RESULTADOS. CONCLUSÕES}

Analisando os dados que nos são dados a interpretar, deparamos primeiramente com uma situação que não é novidade, os rapazes apresentam melhores desempenhos motores do que as raparigas, nas provas de força e velocidade.

Os alunos de Moncorvo apresentam um padrão ligeiramente superior nos testes de Força Superior, "Bola 2Kg" e "lançamento de bola de Hóquei".

Os alunos de Gaia apresentam valores padrão superiores no teste de flexibilidade "Sit and Reach".

Foram detectadas inúmeras correlações positivas e negativas que necessitam de confirmação através de mais dados.

Ao realizarmos este estudo, pretendemos caracterizar os níveis de aptidão física dos jovens escolares de dois Concelhos distintos, um sofrendo da interioridade e outro bastante urbano, uma vez que não existem estudos em Portugal deste género, com qualquer tipo de informação a este respeito.

No que se refere às implicações para a investigação futura, o estudo realizado, pelas suas próprias características, e consequentes limitações, comprovou a necessidade de se investigar mais, muito mais, neste domínio (aptidão física) de forma a contemplarem as propostas sugeridas por Cruz (1994): a) investigação naturalística, em contextos reais, e recorrendo a abordagens multivariadas e multidimensionais (com diferentes métodos], de forma a permitir generalizações científicas; b] estudo dos melhores e piores alunos, que gostem ou não de Educação Física em situação de início de ano lectivo, meio do ano lectivo e final de ano 
lectivo; c) investigação de natureza prospectiva e longitudinal, ao longo do tempo.

Temos consciência que este trabalho por si só, como indicador, é insuficiente, necessário será criar uma base de dados maior, correlacionar outro tipo de factores, sócio-económicos, motivacionais, aferindo os niveis de stress e ansiedade e os seus condicionalismos na aula de Educação Física, caracterização antropométrica mais detalhada.

Mediante essa avaliação, será decisivo para os profissionais, uma melhor selecção e escolha dos critérios, conteúdos e objectivos da aula de Educação Física, no sentido de dotar os jovens de hoje, homens de amanhã, de uma qualidade de vida superior, através de uma regulação efectiva da sua aptidão física e coordenação motora.

\section{BIBLIOGRAFIA}

1. Almeida, C., Aptidão Física, Estatuto Sócio-Económico e Medidas Antropométricas da População Escolar do Concelho de Lamego. Estudo em crianças e jovens de ambos os sexos dos 10 aos 16 anos de idades., in Faculdade de Ciências do Desporto e Educação Física. 1998, Porto: Porto.

2. Baecke, J., J. Burema, and J. Frijters, A short questionnaire for the measurement of habitual physical activity in epidemiological studies. Am J Clin Nutr, 1982. 36(5): pp. 936-942.

3. Baumgartner, T. A., Measurement Concepts in Physical Education and Exercise science. M.J. Safrit e T.M. Wood ed, ed. N.-R.M. Reliability. 1989, Champaign, Illinois: Human Kinetics Books. 45-72.
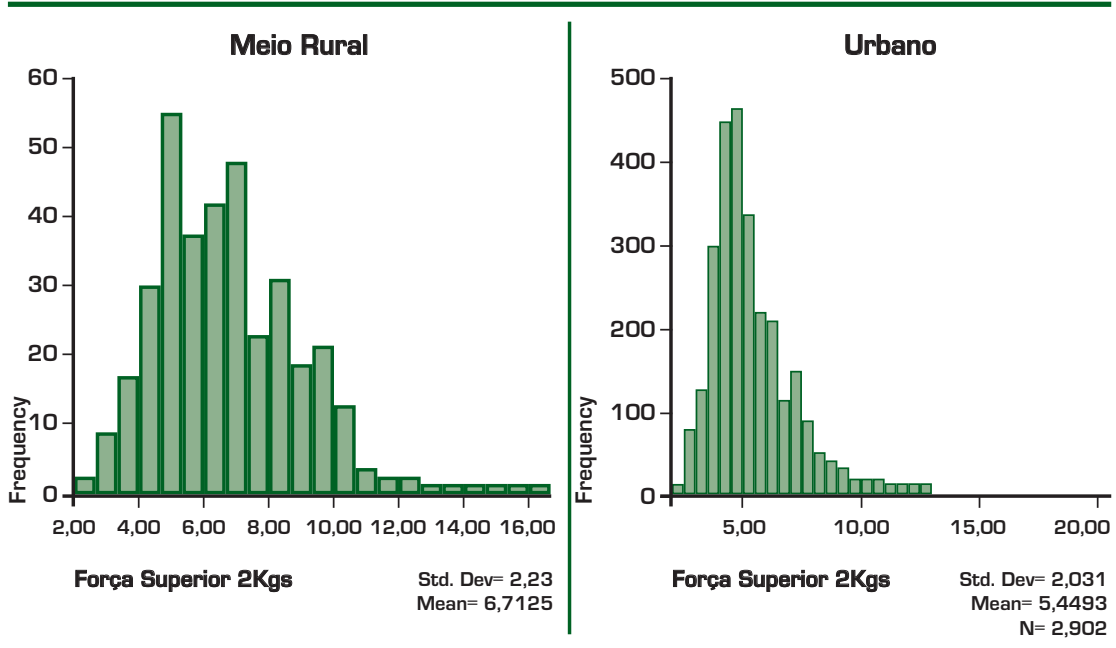

FIGURA5 Comparação de "Força Superior 2Kg".

4. Borms, J., Exercício Físico, Aptidão Física e o Novo Paradigma da Saúde, J.B.e.A. Marques, Editor. 1991, FCDEF: Porto. pp. 111-118. 5. Dâmaso, M.D., Aptidão Física e Indicadores Antropométricos da população escolar do Distrito de Castelo Branco. Estudo em Criancas e Jovens dos 10 aos 14 anos de idade Praticantes de Desporto Escolar, in FCDEF. 1997, Universidade do Porto: Porto.

6. Fredriks, A.M., et al., Body index measurements in 1996-7 compared with 1980. Arch. Dis. Child., 2000. 82(2): pp. 107-112

7. L'Europe, C.d., ed. Eurofit - Test Européen d'Aptitude Physique. Deuxième Edition ed. 1993, Conseil de L'Europe: Strasbourg.

8. Malina, R.M. and P.T. Katzmarzyk, Validity of the body mass index as an indicator of the risk and presence of overweight in adolescents. Am J Clin Nutr, 1999. 70(1): pp. 1315-136.

9. Prista, A., et al., Anthropometric indicators of nutritional status: implications for fitness, activity, and health in school-age children and adolescents from Maputo, Mozambique. Am J Clin Nutr, 2003. 77(4): pp. 952-959.
10. Shephard, R.J. and A. Vuillemin, Limits to the measurement of habitual physical activity by questionnaires * Commentary. $\mathrm{Br} J$ Sports Med, 2003. 37(3): pp. 197-206.

11. Suni J.H. , P.O., S.I Miilunpalo, M.E. Pasanen, I.M. Vuori, K, Bos, Health-related fitness test battery for middle-aged adults: Associations with Physical Activity Patterns, in International Journal of Sports Medicine. 1999. pp. 183-191.

12. Tanner, J. and R. Whitehouse, Clinical longitudinal standards for height, weight, height velocity, weight velocity, and stages of puberty. Arch. Dis. Child., 1976. 51(3): pp. 170-179.

13. van Heuvelen, M.J.G., Martin Stevens, and Gertrudis I.J.M. Kempen, Differences in Physical-Fitness Test Scores Between Actively and Passively Recruited Older Adults: Consequences for Norm-Based Classification. Journal of Aging and Physical Activity, 2002. 10(2): pp. 143-159.

14. Yao, M., et al., Field Methods for Body Composition Assessment Are Valid in Healthy Chinese Adults. J. Nutr., 2002. 132(2): pp. 310-317. 